\title{
LASER BASED ULTRASONICS: A PRACTICAL TOOL FOR NON DESTRUCTIVE TESTING OF MATERIALS
}

\author{
Anita Shukla, Sumiti Narayan Tewari \\ Department of Basic Sciences and Humanities \\ Pranveer Singh Institute of Technology, Kanpur, 209305, India \\ shukla.anita27@gmail.com \\ sntphy@gmail.com
}

\begin{abstract}
Mechanical properties can be determined for all types of materials whether metals, ceramics, polymers, or composites. The material is hence used for specific purpose based on its property. Selection for an appropriate material and treatments for a particular application requires material testing. A material testing helps us to understand and compute whether a specific material is suitable for a particular application. A wide range of materials are available in market for various uses. The choice of material to the most appropriate selection for the intended use can be made easier with the help of material testing. In material characterization, the analysis of material properties and composition is useful in failure investigation and other problems, and in validating manufacturing processes. There are various testing methods available to test the materials. They are classified into two categories destructive testing and non-Destructive Testing (NDT). Present work focuses on superiority of NDT over destructive method and discusses about novel NDT material testing technique called Laser Based Ultrasonics (LBU) technique which is one of the NDT methods for testing and material characterization.
\end{abstract}

Keywords: Mechanical properties, Destructive testing, Non Destructive Testing, Laser Based Ultrasonics, material characterization.

\section{Mathematics Subject Classification 00A79}

\section{INTRODUCTION}

Non-destructive testing (NDT) is an analysis technique which is widely used to evaluate the properties of a material, component or system without causing damage (Louis C, 1995). The term non-destructive examination (NDE), non-destructive inspection (NDI) and nondestructive evaluation (NDE) are the words used in same context to describe this technology (Hellier C,2003) . NDT is highly valuable technique as it does not affect or alter the material under inspection. It saves both money and time in product evaluation, troubleshooting, and research. The quality control of the products, failure analysis or prevention of the engineered systems in service requires the feature of NDT in which evaluation of engineering materials or structures is done without impairing their properties. Only Non-destructive testing (NDT) allows inspecting or measuring the materials or structures without destroying their surface texture, product integrity and future usefulness. NDT is a tool that can be used to locate flaws 
and failures of a material very accurately that might otherwise be the reason for planes to crash, pipelines to burst, fail to reactors, trains to derail, and lots of less noticeable but irreparable losses.

For testing trained operating skilled staff is required. The interpretation of test results accurately may be difficult as the results can be subjective also. These methods can be employed on metals, plastics, defective welds and flaws that could lead to premature failure. The commonly used NDT techniques (3) are Acoustic Emission Testing (AE), Electromagnetic Testing (ET), Guided Wave Testing (GW), Ground Penetrating Radar (GPR), Laser Testing Methods (LM), Leak Testing (LT), Microwave Testing, Liquid Penetrant Testing (PT), Magnetic Flux Leakage (MFL), Magnetic Particle Testing (MT), Neutron Radiographic Testing (NR), Radiographic Testing (RT), Thermal/Infrared Testing (IR), Ultrasonic Testing (UT), Vibration Analysis (VA) and Visual Testing (VT). These techniques have their own capabilities and limitations. Laser Based Ultrasonics is amongst the most promising methods within the several NDT-NDE methods available.

\section{SUPERIORITY OF NON-DESTRUCTIVE TESTING OVER DESTRUCTIVE TESTING}

Non-destructive testing (NDT) is the technique by use of which one can inspect, test, or evaluate materials, components or assemblies.NDT is a novel technique as without destroying the integrity of the part or system this could be used for discontinuities, or differences in characteristics.After the completion of the test the parts or material can still be used. Their utility remains same. NDT is the best effective method to find out the discontinuities and differences in material characteristics.

Destructive tests are usually performed test specimens made for that purpose or some time these are performed on the specimen taken as representative of same material. They are done in laboratories, workshops or training centres and can be chemical or mechanical in nature (Daviest SJ et al.1993). The merits of NDT over destructive testing have been enlisted in Table 1.

Table 1: The Merits of NDT over Destructive Testing

\begin{tabular}{|l|l|}
\hline \multicolumn{1}{|c|}{ NON DESTRUCTIVE TESTING } & \multicolumn{1}{c|}{ DESTRUCTIVE TESTING } \\
\hline $\begin{array}{l}\text { Non-destructive tests may often be applied to } \\
\text { parts in service assemblies without } \\
\text { interruption. There is no loss of serviceable } \\
\text { parts. }\end{array}$ & $\begin{array}{l}\text { Destructive tests are not convenient to apply } \\
\text { to parts in service because the service is } \\
\text { interrupted and there is always possibility of } \\
\text { loss of serviceable parts. }\end{array}$ \\
\hline $\begin{array}{l}\text { It is a healthy method as the parts of very } \\
\text { high material or fabrication costs are not lost. } \\
\text { Hence, it can be employed for repeated } \\
\text { testing during production or service. }\end{array}$ & $\begin{array}{l}\text { It not a healthy method as during the } \\
\text { and the cost or replacing the costly parts } \\
\text { destroyed is unaffordable. Hence, it cannot } \\
\text { be employed for repeated testing during } \\
\text { production or service. }\end{array}$ \\
\hline
\end{tabular}


The tests could be done directly upon the specimen actually used in service. Hence, there is no difference between the readings taken between the specimen tested and those used in service.

Number of non-destructive tests could be applied to the different parts of the specimen in order to measure different properties.

It could be done with the specimens having typical geometries.

Tests can be made on every unit to be used in service if economically justified.
The tests could not be done directly upon the specimen actually used in service. Hence, there is a gap between the readings taken between the specimen tested and those used in service

A single destructive test may measure only one or a few of the properties that may be critical under service conditions.

It is not feasible with the specimens having typical geometries.

Tests can be made on only a fraction of the production lot to be used in service.

\section{LASER BASED ULTRASONICS}

LBU (Laser Based Ultrasonics) is a technique that uses lasers to generate and detect ultrasonic waves. It is a non-contact technique that is used for materials characterization along with the measurement of materials thickness and flaw detection. Laser generation assembly and detection laser are the basic components of a laser-ultrasonic system.

\subsection{Laser Generation of Ultrasonics Waves}

Of all the properties of laser light, the properties that are of interest in Laser Based Ultrasonics are its high intensity and directionality. In this technique, a laser light is used to generate high frequency ultrasound. This is accomplished by illuminating a sample with a short laser pulse. A high intensity laser pulse when impinged upon a target surface creates a stress field either by localized heating leading to the thermal expansion of the surface area around the laser spot or by causing ablation (pulling the atoms out of their lattice sites) (Scruby CB, and Drain LE,1990).

Absorption of the incident pulse energy and the associated temperatures gradients induces a rapidly changing strain field. This strain field, in turn radiates energy as elastic (ultrasonic) waves. At low pulse power, this is an entirely Thermoelastic process resulting in no damage to the sample. The effect is similar to what would be expected from a mechanical impact; i.e. a travelling acoustic wave is generated that propagates both through and along the sample, producing very small disturbance in the surface when it reaches a boundary. This disturbance of the surface due to the wave that reached it can be picked up by various detection methods to be analyzed so that information about the material of the sample under study can be obtained. When the generation of ultrasound using lasers is based upon the thermal expansion due to heating caused by the laser energy then the laser power regime is called Thermoelastic regime (Murray TW and Wagner JW,1998) and is shown in Figure 1. 


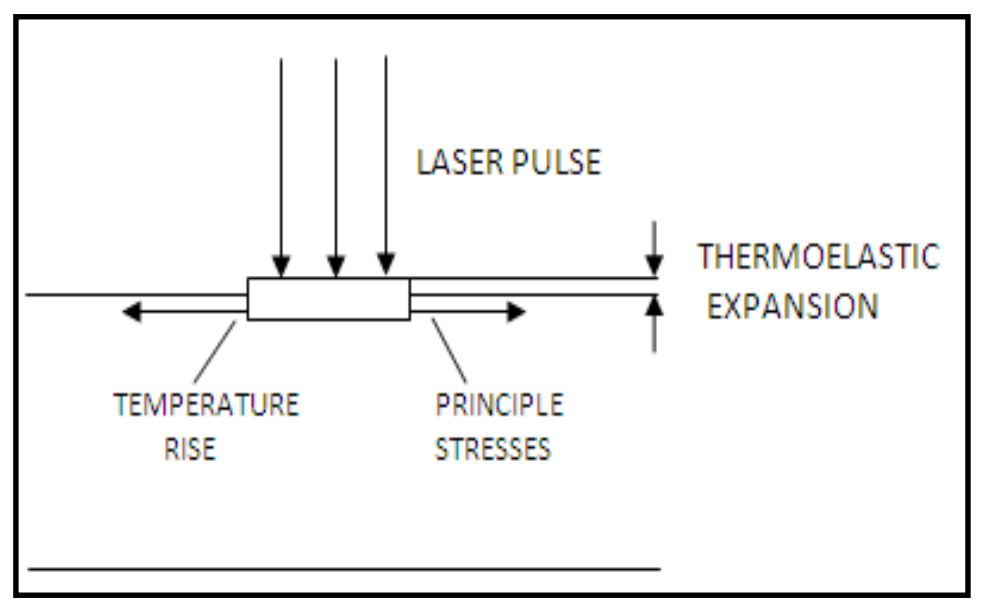

Fig. 1 Thermoelastic regime

If the laser power is such that it can supply energy to the particles beyond their binding energies they get pulled out of the lattice. This process is known as ablation. During ablation process some material is evaporated from the surface and ultrasound is generated by the recoil effect of the expanding material that got evaporated. This power regime is called ablation regime (Haghighi V and Malekmohamadi S,2016) and is shown in Figure 2. In the ablation regime, formation of plasma happens just above the material surface and expansion of plasma makes a considerable contribution to the ultrasonic generation. The frequency content of the generated ultrasound is partially determined by the frequency content of the laser pulses with shorter pulses giving raise to generation of higher frequencies. For very high frequency generation (up to $100 \mathrm{GHz}$ ) femto second lasers are used.

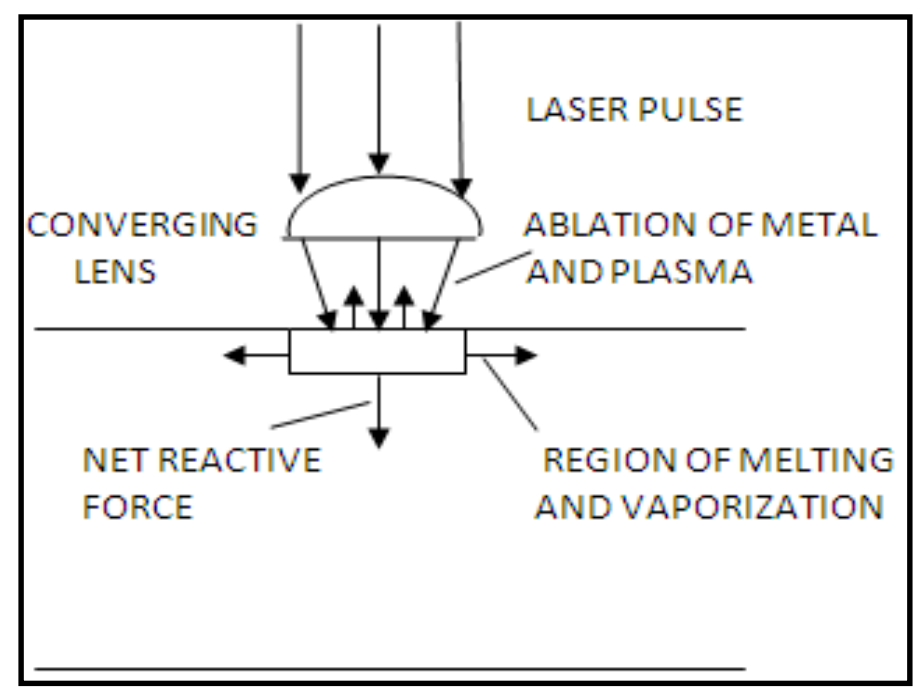

Fig. 2 Ablation regime

Both the thermoelastic and ablation stresses make the particles of the target material to vibrate giving rise to the generation of mechanical waves with frequencies in the ultrasonic region. Laser based ultrasonics (LBU) has proved to be a useful tool for material characterization as well as many other engineering applications. Figure 3 shows the pictorial 
representation of laser generation and detection of ultrasonic waves. This is the configuration used in the present work.

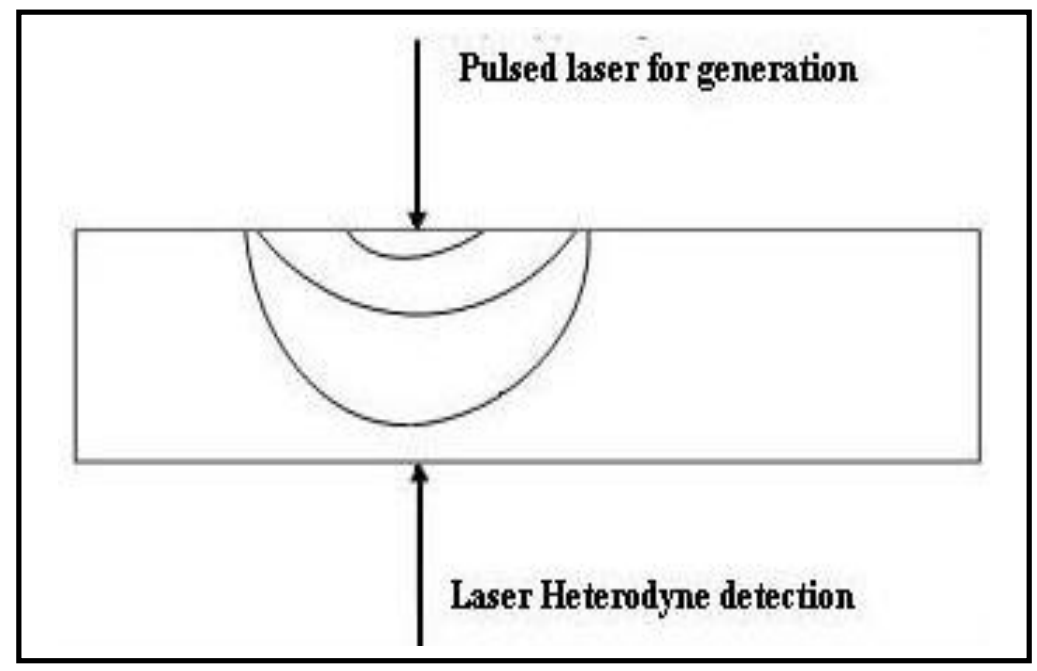

Fig. 3 Laser generation and detection of ultrasonic waves

The ultrasonic waves generated due to the stress applied on one surface, by the impinged laser pulse or by any other method, travel into the medium and undergo reflection/transmission when they encounter an interface of two media, like surface of the material under study and air. The reflected waves travel in accordance with the Snell's laws and reach the original surface where the laser light is impinged. The experimentalists can either study the transmitted waves or the reflected waves to carry out studies on the materials. Different methods of detection can be adopted to detect these waves. One can either use a piezoelectric transducers or a $(\mathrm{Cw})$ laser heterodyne receiver for detection of these waves. The laser-based ultrasonics is the technique, which does not require any mechanical contact with the material under study therefore, this techniques is very attractive in the field of Non Destructive Evaluation (NDE) of materials. The association of laser generation with the optical detection like laser heterodyne detection provides a completely remote ultrasonic system.

LBU techniques for generation and detection of the ultrasound are very different from those using transducers. Lasers can generate and detect the full complement i.e. various types of ultrasonic waves. Laser ultrasonic generation and detection provides significant advantages for in-process inspection.

\subsection{Laser Detection of Ultrasonics Waves}

Optical interferometer (Monchalin JP and Heon R, 1986), is a very sensitive way of measuring displacements, but to be practicable for general use, it requires a highly monochromatic source of light and thus the use of lasers is virtually essential. Interferometers for the detection of ultrasonic movements of waves may be divided into two main types. In the first type, light scattered from a surface is made to interfere with a reference beam, thus 
giving a measure of optical phase and hence instantaneous surface displacement. The second type of interferometer makes use of interference between a large numbers of reflected beams. In order to detect the changes in the frequency of scattered or reflected light this highresolution optical spectrometer is designed. The output obtained is dependent on the velocity of the surface. The first type is the more widely used and the most practical at lower frequencies and with reflecting surfaces. The second type offers a potentially higher sensitivity with rough surfaces, particularly at higher frequencies.

\section{MERITS OF LASER BASED ULTRASONICS}

Laser based ultrasonics is a remote and non-contact method and is preferred over other methods of ultrasonic generation.tis has been found advantageous for testing metals, composite materials, ceramics and liquids. It can remotely and rapidly inspect curved surfaces and typical geometries. It can be used in hostile environments and at high temperatures. Laser UT is fast and effective on rough surfaces. It can function effectively in a factory environment. It ideally suits many applications that are not possible with conventional ultrasonic testing.

\section{CONCLUSIONS}

The accuracy and flexibility of laser based ultrasonics has made it an attractive new option in the non destructive testing market. In this method as the incident energy is due to an electromagnetic radiation in the form of a beam, the beam can readily be steered, focused and expanded as and when the need arises. This method also has the advantage of generation and detection of high-frequency ultrasonic waves which in turn greatly increase spatial resolution and signal-to-noise ratio over contact ultrasonic methods. Laser based ultrasonics is finding more and more applications in many fields of study. It is being widely used in the measurement of the dimensional properties such as thickness, Composition including impurity levels, homogeneity and segregation, Micro structural properties including grain size, texture, number and distribution of phase present, Mechanical properties such as strength, ductility, magnitude of residual stresses ,surface properties like roughness, surface treatment quality, coating quality and thickness, Presence and size of all defects and discontinuities such as cracks, inclusions, porosity etc.

\section{REFERENCES}

[1] Louis, C. (1995) Nondestructive Testing ( A S M International, The United States of America) $3^{\text {rd }}$ Edn.

[2] Hellier, C. (2003) Handbook of Nondestructive Evaluation (ASNT, McGraw-Hill) $2^{\text {nd }}$ Edn.

[3] Introduction to Nondestructive Testing. [Accessed 2022 Feb 14] https://www.asnt.org/MajorSiteSections/About/Introduction_to_Nondestructive_Testing.aspx [4]Daviest, S.J., Edward, C., Taylor, G.S., Palmer, S.B. (1993) Laser-generated ultrasound: its properties, mechanisms and multifarious applications. Journal of Physics D: Applied Physics.26:329-348. 
[5] Scruby, C.B., Drain, L.E. (1990) Laser Ultrasonics, Adam Hilger, Philadelphia, New York. pp 243-358, 76-78

[6] Murray T.W., Wagner, J.W. (1998) Thermoelastic and Ablative Generation of Ultrasound: Source Effects.

Review of Progress in Quantitative Nondestructive Evaluation.17:619-625.

[7] Haghighi, V., Malekmohamadi, S. Detection of laser generated ultrasonic waves in ablation regime by plane and confocal Fabry-Perot interferometers: Simulation.Proceedings of the3rd International Iranian NDT Conference;. 2016, Tehran, Iran.

[8] Monchalin JP, Heon R (1986) Laser ultrasonic generation and optical detection with a confocal Fabry-Perot interferometer. Materials Evaluation.44:1231-1237.

[9] Xiaoyu Yang.,Erik Verboven., Bing-fengJu., Mathias Kersemans

Comparative study of ultrasonic techniques for reconstructing the multilayer structure of composites, NDT \& E International

Volume 121, July 2021, 102460 